\title{
Influence of measurement noise on the PSO based localization of an impact occurring on elastic plates
}

\author{
A. El-Bakari ${ }^{1}$, A. Khamlichi $^{2}$, R. Dkiouak ${ }^{1}$ \\ ${ }^{1}$ Mechanics and Civil Engineering Laboratory, Faculty of Sciences and Techniques, Tangier 91001, Morocco \\ ${ }^{2}$ Systems of Telecommunications and Detection Laboratory, Faculty of Sciences, Tetouan 93030, Morocco
}

\begin{abstract}
This paper presents the application of particle swarm optimization algorithm to reconstruct the characteristics of impacts occurring on homogeneous and isotropic elastic plates and which cannot be reduced to a concentrated force. Solution of this inverse problem was achieved through minimizing a fitness function defined in terms of the root mean square error between the measured and the calculated responses. This included information provided by numerous sensors. The impacting force was assumed to result from uniformly distributed pressure acting over a rectangular patch. Use was made of the reciprocity theorem to decouple the localization problem from the deconvolution part intended to reconstruct the pressure time signal. The particle swarm optimization based model was found to be highly efficient in finding the impact zone location. Focus was done on the robustness aspect of force impact localization when an additive white noise is assumed to perturb response measurements.
\end{abstract}

\section{Introduction}

Structures can be subjected to impacts from various sources. Accidental impacts may cause considerable damage and threaten their integrity. During impacts, simple examination of apparent traces on the structure surface is not sufficient because the damage may be invisible and deep inside the core of the structure [1]. The inspection by experimental means to assess damage that has occurred after an impact event uses specific diagnosis techniques which are too expensive, like for example Xray imaging, tomography, etc.

Identifying the characteristics of the force generated by impact can be used to better know the residual health of structures at risk of impacts, and thus enabling to reduce hugely the required experimental effort [2,3]. In case of simple linear elastic structures with homogeneous geometric and material properties such as beams or plates, identification of impact characteristics can be implemented through using a structural model [4,5]. This can be constructed analytically, by means of the finite element method or by experimental identification procedures. When the impact can be assumed as being punctual and the impact location is known, the impulse response functions between the impact point and the sensors placed at known positions, allows by using a regularized deconvolution technique to reconstruct the force signal [6-9]. When the point of impact is unknown, the inverse formulation uses a minimization technique between the measured and calculated responses to identify the impact characteristics in two steps: at first point location and then force time signal reconstruction [10].

If now the impact is not associated to a concentrated force, the problem is more complex because it involves identifying a distribution of pressure and not only a single force. Even if the pressure can be considered to be uniformally distributed over the domain, new parameters that represent the extent of the impact zone appear in the problem. These are implicit in nature in contrast of the force discrete time components which enter the problem explicitly. To operate minimization between the theoretical model and measurements, an adequate algorithm should be used.

The Particle Swarm Optimization (PSO) modeling is well suited for this kind of implicit optimization problems. PSO is a population based stochastic optimization technique which was developed initially by Kennedy and Eberhart [11]. The underlying biological metaphor for developing PSO algorithm is inspired by social behavior of bird flocking or fish schooling. PSO based techniques has become popular optimization method as they often succeed in finding the best optimum by global search in contrast with most other common optimization algorithms. In comparison for example with the dynamic programming, PSO allows the users to get the sub-optimal solution where dynamic programming cannot. As the localization problem is associated to an impact, which is not represented by a single concentrated force, it contains a lot of unknown variables. PSO can be 
used to handle with grand effectiveness this kind of situation. Furthermore the rate of convergence of PSO based algorithms is known to be fast, this constitutes in structural health monitoring problems a huge advantage as predictions can be made more promptly.

In this work, a PSO based technique is applied in order to solve the localization problem of an impact occurring on elastic rectangular plate like structures which are subjected to impacts that can be represented by a proportional pressure exerted on a rectangular patch. Performance of the PSO algorithm is assessed in terms of noise perturbations that could possibly affect response measurement as provided by some sensors implemented on the plate structure. White noise with a given level is assumed to modify these observation measurements. The objective function to be minimized and the constraints are derived as function of the discrete time responses provided by all possible pairs of sensors while left multiplied by the dual Toeplitz like time transfer matrices. These last are expressed in terms of the conjectured location of the impact zone centre as well as the parameters giving its extent. A parametric study dealing with the effect of the measurement noise on the PSO calculated solution is performed in the case where a total number of five sensors are used.

\section{Formulation of the localization problem}

We consider a rectangular plate as shown in figure 1 which has the dimensions $a, b$ and $e$ representing respectively the length, width and thickness. It is assumed to be simply supported on all its boards. The plate is assumed to be made of a homogeneous and isotropic elastic material having Young's modulus $E$, Poisson's ratio $v$ and density $\rho$.

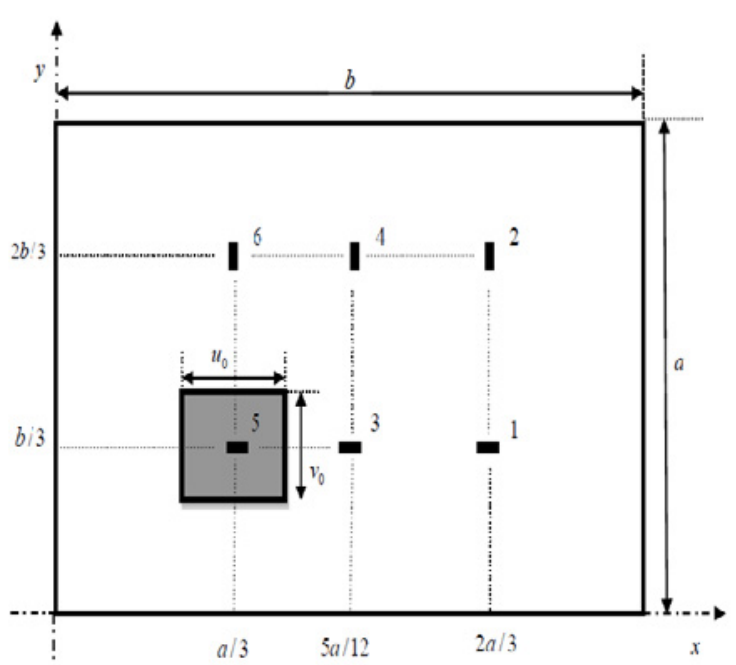

Fig. 1. Simply supported rectangular plate showing the loading patch characteristics and the considered strain gauges locations and orientations.

The applied force modeling impact is assumed to result from pressure that is uniformly distributed over a rectangular patch of the plate. The dynamic response in terms of displacement is considered to be measured at some points belonging to the set of marked points with labels varying between 1 and 6 as shown on figure 1 .

\subsection{Fitness function}

The problem of locating the impact zone having the form of a rectangular patch, requires identifying the impact zone centre position $x_{0}, y_{0}$ and parameters $u_{0}, v_{0}$ defining the extent of the impact zone, which are respectively the dimensions along the $x$ axis and the $y$ axis of the patch. Using the Toeplitz like time transfer matrix of the system, the displacement responses measured by strain sensors placed in points having respectively the coordinates $\left(x_{i}, y_{i}\right)$ can be expressed in the following form

$Y_{i}=H_{i}\left(x_{0}, y_{0}, u_{0}, v_{0}\right) P$

$Y_{j}=H_{j}\left(x_{0}, y_{0}, u_{0}, v_{0}\right) P$

with $\quad H_{i}\left(x_{0}, y_{0}, u_{0}, v_{0}\right)=H\left(x_{0}, y_{0}, u_{0}, v_{0}, x_{i}, y_{i}\right)$ and $H_{j}\left(x_{0}, y_{0}, u_{0}, v_{0}\right)=H\left(x_{0}, y_{0}, u_{0}, v_{0}, x_{j}, y_{j}\right)$.

The commutative property that results from MaxwellBetti theorem applicable to all linear elastic systems, yields as shown in [8-10] to the following relation.

$$
H_{j}\left(x_{0}, y_{0}, u_{0}, v_{0}\right) Y_{i}=H_{i}\left(x_{0}, y_{0}, u_{0}, v_{0}\right) Y_{j}
$$

The interest of this relation is that it does not depend on the time history of the applied pressure $P$. It enables then total decoupling of the problem of localization from that of pressure signal reconstruction. The impact characteristics can like this be identified in two steps. In the first step, the impact location zone is determined. Then, in the second step pressure time signal is obtained through a classical regularized deconvolution technique, like that based on the generalized singular value decomposition theorem [6].

The four parameters that define the impact location $x_{0}, y_{0}, u_{0}$ and $v_{0}$ can be found by minimizing the following fitness function.

$$
\left(x_{0}, y_{0}, u_{0}, v_{0}\right)=\underset{(x, y, u, v)}{A \operatorname{rg} \min }[\phi(x, y, u, v)]
$$

with

$$
\phi(x, y, u, v)=\sum_{\substack{i=1 \\ j=1 \\ j \neq i}}^{N_{s}} \alpha_{i j}\left\|H_{j}(x, y, u, v) Y_{i}-H_{i}(x, y, u, v) Y_{j}\right\|^{2}
$$

where $N_{s} \geq 2$ denotes the number of sensors used and $\alpha_{i j}$ are weights that are introduced to avoid the trivial solutions of equation (3). The following expressions can be taken for the coefficient $\alpha_{i j}$

$$
\alpha_{i j}=\frac{1}{\left\|H_{i}(x, y, u, v)\right\|^{2}+\left\|H_{j}(x, y, u, v)\right\|^{2}}
$$


In the presence of measurement noise, the perturbed fitness function takes the following form

$$
\phi(x, y, u, v)=\sum_{\substack{i=1 \\ j=1 \\ j \neq i}}^{N_{s}} \alpha_{i j}\left\|H_{j}(x, y, u, v) Y_{i}-H_{i}(x, y, u, v) Y_{j}\right\|^{2}
$$

with

$\tilde{Y}_{i}=\left(1+v_{i} z_{k}\right)_{i}\left(Y_{k}\right)_{i}$ and $\tilde{Y}_{j}=\left(1+v_{j} z_{k}\right)_{j}\left(Y_{k}\right)_{j}$

where $z_{k}$ is a random number belonging to the interval $[-1,1]$ and $v_{i}$, respectively $v_{j}$, designates the noise level present in measurement delivered by sensor $i$, respectively sensor $j$.

To ensure uniqueness of the solution and also to accelerate the convergence rate, the minimization of the functional $\phi$ is subjected to the following constraints.

$0.1 a \leq x_{0} \leq 0.9 a$

$0.1 b \leq y_{0} \leq 0.9 b$

$0.1 a \leq u_{0} \leq 0.5 a$

$0.1 b \leq v_{0} \leq 0.5 b$

\subsection{Particle Swarm Optimization}

The problem defined by equations (4), (5), (6), (7) and (8) takes the form of a nonlinear mathematical program for which it is not easy to give explicitly the objective function in terms of the unknowns. To solve it, an evolutionary algorithm based on PSO algorithm is used $[11,12]$.

The PSO is a heuristic optimization method that mimics the social behavior. Its implementation needs to specify the protocol of cooperation and competition among the potential solutions. In this technique, the initial population of particles of the objective function to be minimized is chosen randomly. In the process, each particle is given an index $i$ ranging from 1 up $N_{p}$. It is assumed to occupy the position $X_{i}(t)$ in the space of unknowns and to have the velocity of displacement $V_{i}(t)$. For each generation $t$, the value of the objective function in each position $X_{i}(t)$ is calculated and the following updating rules are applied

$V_{i j}(t+1)=w V_{i j}(t)+c_{1} r_{1 j}(t)\left(P_{i j}(t)-X_{i j}(t)\right)$

$+c_{2} r_{2 j}(t)\left(g_{j}(t)-X_{i j}(t)\right)$

$X_{i j}(t+1)=X_{i j}(t)+V_{i j}(t)$

with $i=1, \ldots, N_{p}$ and $j=1, \ldots, 4$, where $w$ is the coefficient of inertia, $c_{1}$ and $c_{2}$ designate the PSO acceleration coefficients, $r_{1}$ and $r_{2}$ are two random numbers drawn uniformly at each iteration in the interval $[0,1], P_{i}=\left(P_{i 1}, \ldots, P_{i d}\right)$ is the best position reached by the particle having index $i$ and $g_{j}$ the best overall position reached by all particles of the considered swarm.

The term $w V_{i j}(t)$ represent the physical component of displacement; the particle tends to follow its current direction of travel. The term $c_{1} r_{1 j}(t)\left(P_{i j}(t)-x_{i j}(t)\right)$ is the cognitive component of displacement; the particle tends to be attracted by the best site in which it has already passed. The term $c_{2} r_{2 j}(t)\left(g_{j}(t)-x_{i j}(t)\right)$ is the social component part of displacement; the particle tends to move towards the best position already achieved by its neighbors.

\section{Results and discussion}

The impact zone characteristics considered in the following are given (in $\mathrm{m}$ ) by: $x_{0}=0.0683$, $y_{0}=0.0683, u_{0}=0.0342$ and $v_{0}=0.0342$. The pulse duration considered is equal to $T=6 \mathrm{~ms}$. Figure 2 presents the impact pressure time signal. The maximum pressure is $2 \times 10^{5} \mathrm{~Pa}$.

Fixing the extent of the impact zone to the exact values $u_{0}=0.0342$ and $v_{0}=0.0342$, figure 3 gives the fitness function associated to the configuration defined by the sensors having the following labels: 1, 2, 3, 4 and 6, as function of the impact zone centre coordinates: $\left(x_{0}, y_{0}\right)$. Figure 3 shows clearly that a unique minimum exists in terms of variables $\left(x_{0}, y_{0}\right)$ when $\left(u_{0}, v_{0}\right)$ are fixed to the previous values. The complete fitness function is defined in terms of the four parameters $\left(x_{0}, y_{0}, u_{0}, v_{0}\right)$ and its representation is not possible. The PSO based algorithm should perform search of the minimum in the fourth dimensional space containing all the unknowns of the localization problem in the presence of measurement noise.

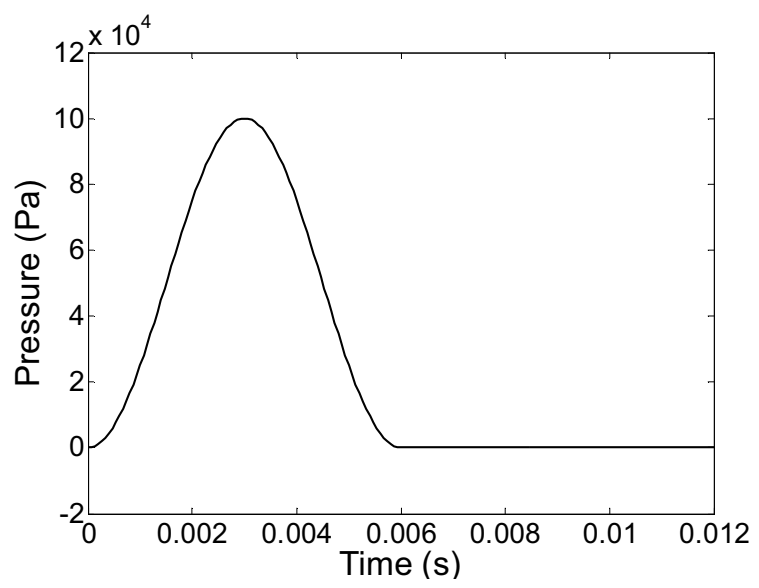

Fig. 2. Pressure signal profile having half-sine form of duration $T=0.006 s$ and $P_{\max }=2 \times 10^{5} \mathrm{~Pa}$ 


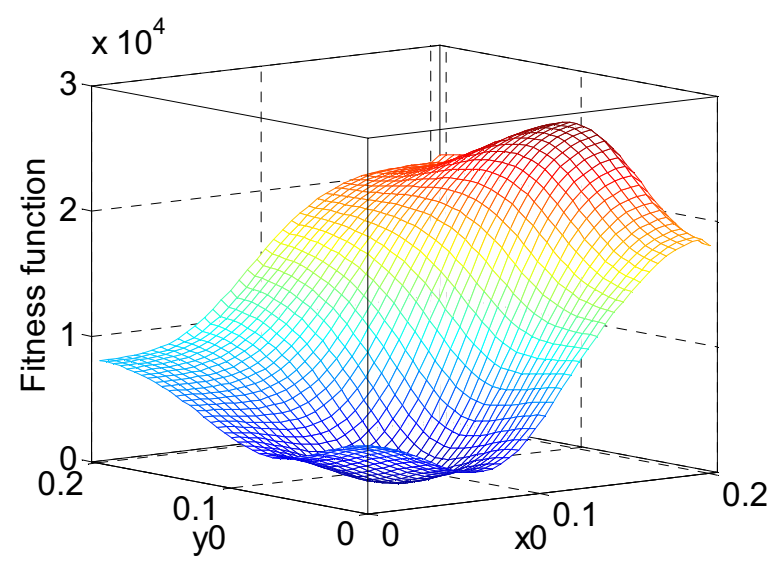

Fig. 3. Surface representing the objective function as function of $\left(x_{0}, y_{0}\right)$ with $u_{0}=0.0342$ and $v_{0}=0.0342$

Using the sensors mentioned above, three noise levels were considered in order to test robustness of the PSO based algorithm. They correspond to the following:

Case 1: noise free case where the noise amplitude is set equal to zero for the five sensors;

Case 2: noise level is fixed at $v_{i}=2 \%$ for the five sensors;

Case 3: noise level is fixed at $v_{i}=5 \%$ for the five sensors.

The parameters used for the PSO were fixed as follows: $w=0.4 ; c_{1}=1.25 ; c_{2}=0.4$ and size population $N_{p}=40$.

Figures $4 \mathrm{a}, 4 \mathrm{~b}, 4 \mathrm{c}$ and $4 \mathrm{~d}$ show the variations of the four parameters $\left(x_{0}, y_{0}, u_{0}, v_{0}\right)$ as function of the iterations performed by the PSO Algorithm. Table 1 gives the relative error at convergence. One can notice that the two noise levels $2 \%$ and $5 \%$ do not disturb significantly the solution in terms of the impact zone center coordinates; the relative error does not exceed $1.6 \%$ in the worst case. However, a stronger disturbance occurred for the parameters which establish the extent of the impact zone $\left(u_{0}, v_{0}\right)$ as the relative error reached $8 \%$.

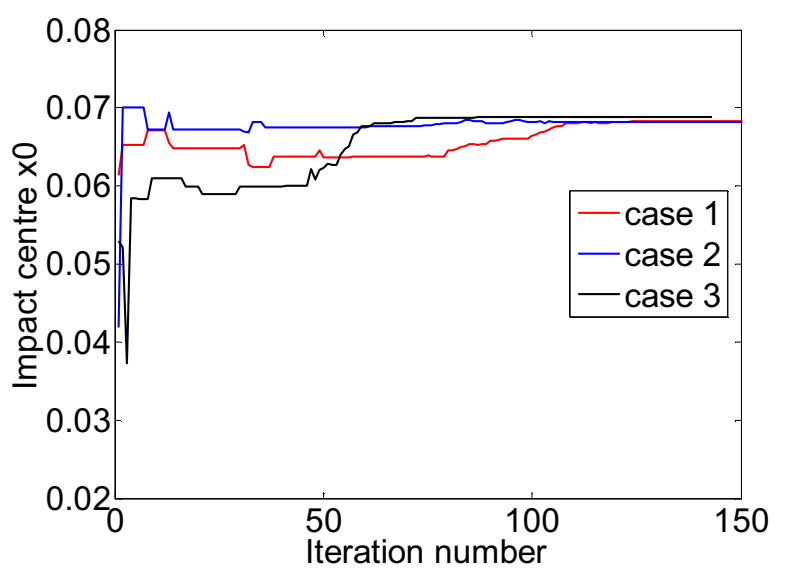

Fig. 4a. Evolution of the impact zone centre abscissa $x_{0}$ as function of the iteration number

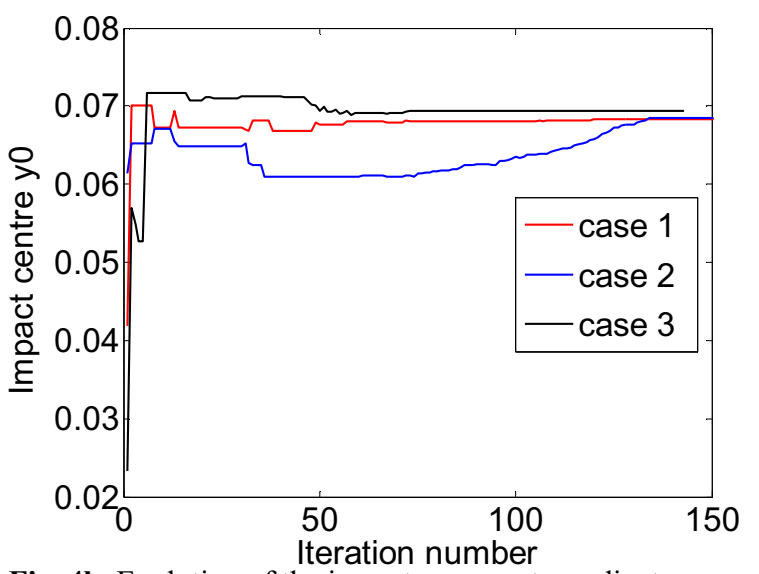

Fig. 4b. Evolution of the impact zone centre ordinate $y_{0}$ as function of the iteration number

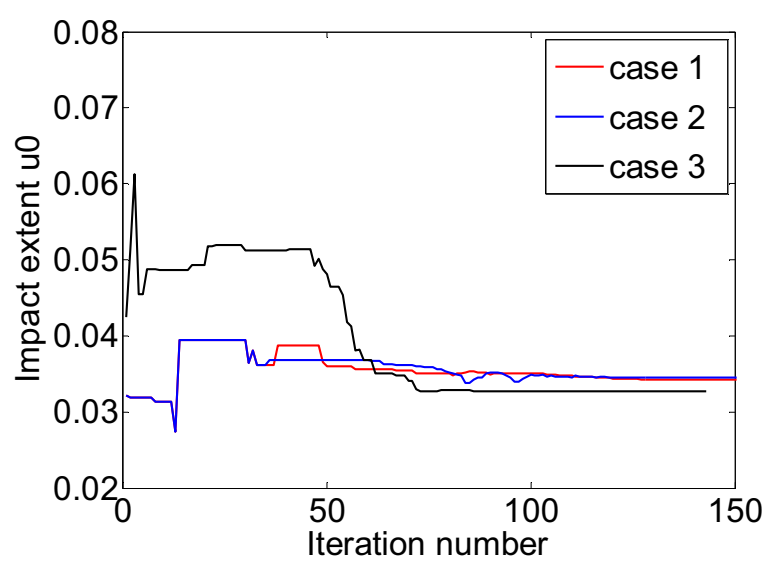

Fig. 4c. Evolution of the impact zone length $u_{0}$ as function of the iteration number

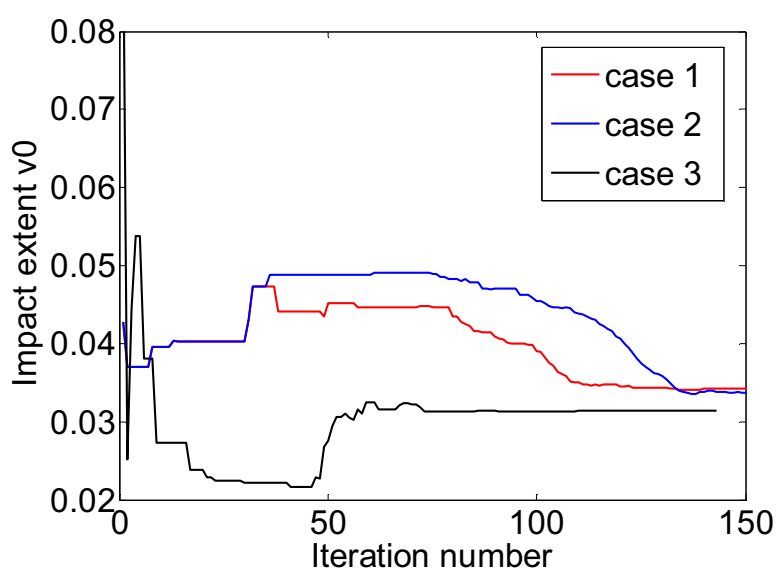

Fig. 4d. Evolution of the impact zone width $v_{0}$ as function of the iteration number

Limiting the acceptable error to a reasonable value of $2 \%$, one can conclude that the noise level $2 \%$ has not perturbed the determination of the impact zone characteristics as the maximum relative error has not exceed $1.35 \%$. 
Table 1. Relative error expressed in \% on the characteristics of the impact zone as obtained after convergence of the PSO for a given noise level

\begin{tabular}{|c|c|c|c|c|}
\hline $\begin{array}{c}\text { Noise } \\
\text { level }\end{array}$ & $x_{0}$ & $y_{0}$ & $u_{0}$ & $v_{0}$ \\
\hline Case 1 & 0 & 0 & 0 & 0 \\
\hline Case 2 & 0.15 & 0.293 & 0.703 & 1.35 \\
\hline Case 3 & 0.713 & 1.61 & 4.4 & 8 \\
\hline
\end{tabular}

\section{Conclusions}

Using decoupling provided by the reciprocity theorem, the localization problem has been formulated as a constrained non linear mathematical program. Solution of this problem was performed by using a particle swarm optimization algorithm. Robustness of this localization procedure to random noise affecting measurement has been achieved in the case where five sensors were used. The algorithm has been found to continue providing the problem solution for low value noise levels not exceeding $2 \%$. With high values of noise, the obtained results at convergence were false.

These results are only valid for the configuration of sensors tested here with the actual system modeled by the Toeplitz like matrix established in the stated particular conditions. Further tests should be performed to draw more complete statement about noise effect on the localization problem.

\section{References}

1. A.G. Miller, D.T. Lovell, J.C. Seferis, Composite Structures, 193-206, 27(1-2) (1994)

2. M. Tracy, F.K. Chang, Part I, Journal of Intelligent Material Systems and Structures, 920-928, 9(11) (1998)

3. M. Tracy, F.K. Chang, Part II, Journal of Intelligent Material Systems and Structures, 929-937, 9(11) (1998)

4. R. Seydel, F.K. Chang, Smart Materials and Structures, 354-369, 10(2) (2001)

5. R. Seydel, F.K. Chang, Smart Materials and Structures, 370-379, 10(2) (2001)

6. P.C. Hansen, Rank-Deficient and Discrete Ill-posed Problems (SIAM, Philadelphia, 1998)

7. N. Hu, H. Fukunaga, S. Matsumoto, B. Yan, X.H. Peng, International Journal of Impact Engineering, 1258-1271, 34(2007)

8. C.S. Yen, E. Wu, Part I, Journal of Applied Mechanics, Transactions of the ASME, 692-698, 62(1995)

9. C.S. Yen, E. Wu, Part II, Journal of Applied Mechanics, Transactions of the ASME, 699-705, 62(1995)

10. A. El-Bakari, A. Khamlichi, E. Jacquelin, R. Dkiouak, Journal of Sound and Vibration, 333(2014)
11. R.C. Eberhat, J. Kennedy, Proceedings of Sixth International Symposium on Micro machine and Human Science, Nagoya, Japan, (1995)

12. M. Clerc, J. Kennedy, IEEE Trans Evol Comput. 6(2) (2002) 